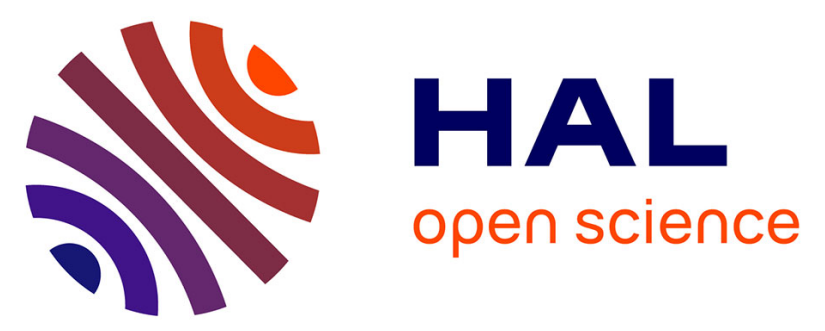

\title{
Inferring bubble volume fraction in a glass melt through in situ impedance spectroscopy measurements
}

\author{
Luiz Pereira, Muriel Neyret, Annabelle Laplace, Franck Pigeonneau, Rafael
}

Nuernberg

\section{- To cite this version:}

Luiz Pereira, Muriel Neyret, Annabelle Laplace, Franck Pigeonneau, Rafael Nuernberg. Inferring bubble volume fraction in a glass melt through in situ impedance spectroscopy measurements. International Journal of Applied Glass Science, In press, 10.1111/ijag.15895 hal-03229456

\section{HAL Id: hal-03229456 \\ https://hal.science/hal-03229456}

Submitted on 19 May 2021

HAL is a multi-disciplinary open access archive for the deposit and dissemination of scientific research documents, whether they are published or not. The documents may come from teaching and research institutions in France or abroad, or from public or private research centers.
L'archive ouverte pluridisciplinaire HAL, est destinée au dépôt et à la diffusion de documents scientifiques de niveau recherche, publiés ou non, émanant des établissements d'enseignement et de recherche français ou étrangers, des laboratoires publics ou privés. 


\title{
INFERRING BUBBLE VOLUME FRACTION IN A GLASS MELT THROUGH IN SITU IMPEDANCE SPECTROSCOPY MEASUREMENTS
}

Luiz Pereira, ${ }^{1,2^{*}}$ Muriel Neyret, ${ }^{1}$ Annabelle Laplace,${ }^{1 * *}$ Franck Pigeonneau, ${ }^{2}$ Rafael Nuernberg ${ }^{1}$

${ }^{1}$ CEA, DES, ISEC, DE2D, Univ Montpellier, Marcoule, France

${ }^{2}$ Centre for Material Forming, CEMEF, MINES ParisTech, PSL Research University, CNRS UMR 7635, CS 10207, Claude Daunesse, 06904, Sophia Antipolis Cedex, France

* luiz.pereira@outlook.fr

** annabelle.laplace@cea.fr

\begin{abstract}
We evaluated the feasibility of inferring the bubble volume fraction $\left(\phi_{b}\right)$ in a melt by employing in situ impedance spectroscopy measurements. This idea stems from the effective medium theory, in which the ionic conductivity of a biphasic material can be determined from the isolated contribution of each constituent phase and their respective volumetric content. Therefore, we investigated the overall electrical conductivity of a borosilicate melt doped with cerium-IV oxide at a constant temperature $(1273 \mathrm{~K})$, in which $\mathrm{O}_{2}$ gas production takes place. To validate the use of this proposed technique, post-mortem bubble volume fraction analyses on samples synthesized following the same procedure were conducted by apparent density and impedance spectroscopy measurements at low temperatures (below $\mathrm{T}_{\mathrm{g}}$ ). A comparison of these post-mortem and in situ techniques is presented. Despite some discrepancies observed between the bubble volume fractions estimated by these different methods, the main results demonstrate the technical viability of this in situ approach. In situ impedance spectroscopy presents advantages in terms of presynthesis duration, sample preparation, and the information is obtained at high temperatures, eliminating the possible influence of the cooling stage.
\end{abstract}


KEYWORDS: bubble, glass melt, ionic conductivity, impedance spectroscopy 


\section{INTRODUCTION}

Bubbled materials can be considered a limiting case composite. ${ }^{1,2}$ This low-density phase may be either beneficial or not, depending on its characteristics and the final material application. This phase usually has a detrimental effect when mechanical or corrosion behaviors are considered. On the other hand, these gaseous inclusions usually decrease the thermal and electrical conductivities of solid and liquid materials. Hence, for insulating systems, these composite materials are considered a useful option. In terms of glass/melt engineering processes, bubbles may affect the overall electrical conductivity of a glass bath, ${ }^{3}$ and the direct induction process could also be affected ${ }^{4}$ because bubbles may negatively affect the thermal and electrical conductivities, resulting in low efficiency and elevated costs.

These bubbled materials have been investigated for decades using several experimental methods. To study a population of bubbles, for example, different techniques have been applied so far. Microscopic techniques have been applied to investigate bubbles in different fields, such as volcanology and glass science. ${ }^{5-7}$ This is a destructive post-mortem approach, in which bubbles are formed in the liquid phase at high temperatures. After cooling, a cross-sectional area of the solidified material was observed. Through this method, a two-dimensional (2D) space is analyzed, and stereological techniques are usually applied to convert 2D bubble features into 3D ones. ${ }^{8-10}$ The microscopic approach, as described in some experimental works, is a laborious and timeconsuming task. ${ }^{5-7}$ High-definition X-ray computed tomography is another robust technique applied to investigate a population of bubbles in melts at high temperatures. ${ }^{11-13}$ Unlike the microscopic approach, this methodology is a non-destructive in situ technique, in which information can be collected simultaneously with the high-temperature experiment. Using this tomography technique, bubble features can be acquired, and owing to sample rotation during data acquisition, 3D information can be obtained during the in situ experiment. Therefore, no stereological data treatment is required. However, the availability of this technique is a major drawback. 
Both post-mortem microscopy and in situ X-ray computed tomography can provide individual information about bubbles, such as bubble size distribution and bubble mean density. However, due to the laborious and time-consuming characteristics of the microscopic technique along with the low availability and cost of the in situ X-ray computed tomography, in this current work, a novel method to infer bubble volume fraction $\left(\phi_{\mathrm{b}}\right)$ in a glass melt is proposed. A material doped with a less conductive dispersed phase, such as bubbles, exhibits a lower overall electrical conductivity. This decay is related to the volumetric fractions based on the effective medium theory. Consequently, high-temperature impedance spectroscopy can be used to determine the bubble volume fraction. Similarly, impedance techniques have been applied to obtain measurements of volume fractions in different multiphase flows. ${ }^{14},{ }^{15}$ In addition, electrical conductivity has also been applied to infer residual glass composition during glass crystallization and study the liquid-liquid phase separation and structural relaxation of glasses. ${ }^{16-19}$

Herein, the electrical conductivity of a borosilicate melt doped with cerium oxide was investigated at a constant temperature $(\mathrm{T}=1273 \mathrm{~K})$. Borosilicate melt compositions constitute the most common nuclear waste conditioning matrices. ${ }^{20}$ Cerium is selected because it is a multivalent element, Ce-(IV) and Ce-(III), and it is generally found in nuclear waste glasses. ${ }^{21}$ Its redox reaction, as given in equation (1), shows that molecular oxygen may be generated, and as a consequence, it may give rise to bubbles. Thus, bubbles might be present in this medium, and from the "effective medium theory," the overall electrical conductivity values can be converted into bubble volume fractions. ${ }^{22}$ The use of high-temperature impedance spectroscopy can constitute an in situ, valuable, and simple way to infer bubble volume fraction, allowing scientists to more easily test new glass compositions. Herein, we use apparent density measurements, postmortem impedance spectroscopy at low temperatures (below $\mathrm{T}_{\mathrm{g}}$ ), and in situ impedance spectroscopy at high temperatures (above $\mathrm{T}_{\mathrm{g}}$ ) to infer the bubble fraction of the studied material. A comparison of all these techniques is performed in order to validate the use of the latter. 


$$
\mathrm{Ce}^{4+}+\frac{1}{2} \mathrm{O}^{2-} \rightleftharpoons \mathrm{Ce}^{3+}+\frac{1}{4} \mathrm{O}_{2}
$$

\section{MATERIALS AND METHODS}

\subsection{Sample description}

Glasses and melts were prepared to verify the use of high-temperature impedance spectroscopy to determine the bubble fraction. They were synthesized by mixing borosilicate glass powder with controlled granulometry $(250-500 \mu \mathrm{m})$ with cerium-IV oxide $(<5 \mu \mathrm{m})$. The former is already synthesized at $1723 \mathrm{~K}$ and is better described by Pflieger et al. ${ }^{23}$ while the latter is supplied by Aldrich and has a chemical purity above $99.9 \%$. The final glass has a $\mathrm{T}_{\mathrm{g}}$ of $804 \mathrm{~K}$, and its final composition is listed in Table 1.

In this study, two glasses were synthesized. The first series consisted of glasses with different bubble fractions, which were first synthesized and then subjected to post-mortem apparent density and low-temperature impedance measurements. The second one can be described as synthesized directly inside the high-temperature impedance measurement system so that the bubble fraction behavior can be monitored using electrical conductivity measurements (Figure 1). It is important to emphasize that both series of glasses have exactly the same composition, and the same synthesis history is sought.

For each sample of the first series, $20 \mathrm{~g}$ of glass was synthesized according to the following procedure. The glass powder was initially mixed mechanically with $\mathrm{CeO}_{2}$ and then placed in a cylindrical alumina crucible (CeraQuest AC20) with an outer diameter, height, and thickness of 30, 40, and $2 \mathrm{~mm}$, respectively. They were melted at $1273 \mathrm{~K}$ for different durations: $20,40,60$, 120, 240, and $360 \mathrm{~min}$, corresponding to different bubble fractions in the glass, ${ }^{7}$ which were subsequently removed from the furnace and cooled to room temperature in air. After synthesis, 
these crucibles were cut in the middle, and parallelepiped samples with thickness of approximately $2 \mathrm{~mm}$ and surface area of approximately $100 \mathrm{~mm}^{2}$ were obtained. Figure 2 provides an optical microscopic image showing the position and orientation of the sample in the crucible. This parallelepiped solid sample was then polished and used for post-mortem apparent density and low-temperature impedance measurements.

The sample of the second group, which was evaluated by high-temperature impedance spectroscopy, was produced in two steps. First, the glass powder was mechanically mixed with the $\mathrm{CeO}_{2}$. Subsequently, we pre-synthesized it by filling the crucible (outer diameter, height, and thickness equal to 30,25 , and $2 \mathrm{~mm}$, respectively) in a muffle furnace at $973 \mathrm{~K}$ with approximately $50 \mathrm{~g}$ of the previous mixture, which was partially added in three steps. After each addition, the crucible was kept in the furnace for 20 min to sinter and create space for the next addition, up to the point where the crucible was almost full. After this achievement, the crucible was removed from the furnace and introduced into the high-temperature impedance measurement system. This filling procedure is required to completely cover the electrodes during the high-temperature experiment and avoid inaccurate measurements. Bubble motion can be neglected in this presynthesis stage because at $973 \mathrm{~K}$, this glass melt is extremely viscous $\left(1.3510^{5} \mathrm{~Pa} \mathrm{~s}\right){ }^{7}$

\subsection{Density measurements}

Density measurements of the bubbled glasses were performed at room temperature using a methodology based on the Archimedean method with water as an immersion liquid and a Mettler AT200 scale. ${ }^{24}$ Measurements were replicated three times for each sample, and using this methodology, the density of a sample can be calculated by:

$$
\rho=\frac{\mathrm{W}_{\mathrm{A}}}{\mathrm{W}_{\mathrm{A}}-\mathrm{W}_{\mathrm{W}}}\left(\rho_{w}\right)
$$


where $\mathrm{W}_{\mathrm{A}}$ is the scale reading with the specimen on the stirrup in the air, $\mathrm{W}_{\mathrm{W}}$ is the scale reading with the specimen on the submerged stirrup, and $\rho_{\mathrm{w}}$ is the water density. The air density is considered to be zero in this case.

Once the densities are obtained, the bubbled sample's glass fraction can be determined by the ratio between the sample's apparent density and a free-bubbled glass sample. Consequently, the bubble volume fraction is obtained by subtracting the glass fraction from the unit. The free-bubbled glass employed as a reference here is that synthesized at $1273 \mathrm{~K}$ for $360 \mathrm{~min}$. The bubble volume fraction of this latter sample, acquired using a post-mortem optical microscopic approach, ${ }^{7}$ was lower than 0.005 . Thus, by selecting the $1273 \mathrm{~K}-360 \mathrm{~min}$ sample as the reference sample for this study, one can compare the results of the different techniques, with a reasonable compromise between the minimization of the experimental time and maximization of accuracy. It is important to emphasize that the same samples used for density measurements were also used in lowtemperature spectroscopy measurements.

\subsection{Low-temperature impedance spectroscopy measurements}

Subsequent to the density measurements, gold electrodes approximately $100 \mathrm{~nm}$ thick were sputtered on both sides of the two oppositely polished faces of glass samples to ensure electrical contact. Impedance spectroscopy measurements were conducted using a Solarton 1260 impedance/gain phase analyzer, over frequency between $1 \mathrm{MHz}$ and $1 \mathrm{~Hz}$, with an applied root mean square $\mathrm{AC}$ voltage of $100 \mathrm{mV}$. A two-electrode cell configuration equipped with flat platinum plates was employed to facilitate efficient electrical contact. Measurements were performed under an air atmosphere and at temperatures below $\mathrm{T}_{\mathrm{g}}$, ranging from 600 to $800 \mathrm{~K}$. The furnace was set to a heating rate of $20 \mathrm{~K}$ per hour, and impedance measurements were continuously performed every $30 \mathrm{~min}$ under heating. Because the impedance spectroscopy measurements took approximately $2 \mathrm{~min}$, the sample variation temperature during the 
measurement was approximately $0.66 \mathrm{~K}$. A thermocouple type $\mathrm{S}$ was placed a few millimeters from the sample to assess the actual temperature of the sample at the time of the measurement.

\subsection{High-temperature impedance spectroscopy measurements}

Impedance spectroscopy measurements were conducted using a Material Mates impedance analyzer over frequency between $1 \mathrm{MHz}$ and $10 \mathrm{~Hz}$, with an applied root mean square AC voltage of $20 \mathrm{mV}$. Measurements were performed in a two-electrode cell adapted from a four-electrode cell described elsewhere. ${ }^{25}$ The two-electrode setup was chosen because the four-electrode setup has two central electrodes that prevent bubbles from freely escaping. The sample pre-synthesized at $973 \mathrm{~K}$ was placed in the high-temperature impedance spectroscopy measurement system and heated at a rate of $30 \mathrm{~K} / \mathrm{min}$ up to $1273 \mathrm{~K}$. The temperature of the crucible took between 20 and 30 min to equilibrate, as evidenced by temperature measurements taken with a type $\mathrm{S}$ thermocouple placed at the bottom of the crucible. Meanwhile, the two plate-like electrodes with dimensions of $12 \mathrm{~mm} \times 8 \mathrm{~mm}$ and $10 \mathrm{~mm}$ away from each other were inserted into the melt. These surface dimensions are similar to the solid samples previously mentioned. Therefore, the present method is sensitive to the spatial average of the bubble volume fraction in the region between the electrodes. The electrode cell is mechanically coupled with a micrometric displacement system, enabling us to detect the surface of the liquid with precision, and therefore, to plunge the electrodes to a reproducible depth. The detection of the liquid surface is based on the contrasting impedance of air and the low impedance of the liquid. The electrode cell was then inserted into the liquid to the immersion depth (approximately $6 \mathrm{~mm}$ below the surface). It is also essential to keep the electrodes at a safe distance from the bottom of the crucible to avoid parasitic current flowing through the crucible wall (at least half of the distance between the electrodes). Once the chosen temperature of $1273 \mathrm{~K}$ was reached with a maximum variation of $1 \mathrm{~K}$ over time, measurements were carried out in air for $720 \mathrm{~min}$. This latter duration is selected to ensure that the bubble-melt resistance does not change considerably from the considered zero-bubble situation (360 $\mathrm{min})$ to $720 \mathrm{~min}$. 


\section{RESULTS AND DISCUSSION}

\subsection{Apparent density}

The glass fraction in the bubbled material can be determined by the ratio between the apparent density of the sample and the free-bubbled glass sample. Consequently, by subtracting the glass fraction from the unit, the volume fraction was obtained from the following relation:

$$
\phi_{\mathrm{b}}=1-\frac{\rho_{\text {bubbled }}}{\rho_{\text {bubble-free }}}
$$

$\phi_{\mathrm{b}}$ is the bubble volume fraction, while $\rho_{\text {bubbled }}$ and $\rho_{\text {bubble-free }}$ are the densities of the bubble-glass and free-bubbled glass, respectively.

Figure 3 shows the apparent density and bubble volume fraction evolution for the glasses synthesized at $1273 \mathrm{~K}$ for different durations (0, 40, 60, 120, 240, and $360 \mathrm{~min})$, in which the last value, as mentioned above, is used as a free-bubbled glass. Indeed, the density of this sample, when compared to the reference bulk one, indicates that the bubble volume fraction is lower than 0.005. ${ }^{7}$ As shown in Figure 3, a decrease in the apparent density occurs until it passes through a minimum at approximately the 60 min synthesis duration and increases afterward. Consequently, the bubble volume fraction $\left(\phi_{\mathrm{b}}\right)$ behaves inversely, passing through a maximum at the same point and decreasing toward zero at the end. An investigation of the same glass system carried out by our research group and investigated through post-mortem optical microscopy demonstrated similar behavior in terms of the evolution of bubble fraction as a function of time. ${ }^{7}$ In this study, the mechanisms of bubble formation and behavior are extensively discussed. 


\subsection{Low-temperature ionic conductivity}

By considering the post-mortem samples synthesized at $1273 \mathrm{~K}$ under different dwell times, we aim to verify whether the electrical conductivity is sufficiently sensitive to estimate the bubble fraction and whether cerium speciation influences the electrical conductivity. The determination of the bubble fraction based on the electrical conductivity is only reliable if the conductivity of the glass phase remains unaffected by the change in cerium speciation when the bubble fraction evolves. In other words, the conduction mechanism should be the same regardless of the evolution of cerium speciation. As recently demonstrated, cerium reduction from $\mathrm{Ce}$-(IV) to $\mathrm{Ce}$-(III) takes time to reach equilibrium at $1000{ }^{\circ} \mathrm{C}^{7}$ In addition, the cerium oxidation state influences how this element acts in the glass structure, being either a network-former or network-modifier. Thus, cerium speciation may also affect the electrical conductivity of glass..$^{26,27}$

Therefore, we carried out post-mortem impedance spectroscopy at different temperatures to determine the activation energy associated with the conduction mechanisms and evaluated whether the change in the valence state of cerium affects the electrical conductivity of the glassy phase. The impedance responses display a typical ion-conducting behavior, typified by a straight and steep increase in the imaginary part of the impedance (spike) at low frequency, together with the impedance response of the sample (semi-circle) at higher frequencies (Fig. S1). This lowfrequency response is commonly denoted as a polarization effect and is given by the blockage of mobile ions at the interface glass/metallic electrodes. ${ }^{28}$ A suitable equivalent circuit is employed to fit the impedance data and extract the resistance of the sample (Fig. S1). The ionic conductivity of each sample was then calculated according to the resistance and their respective dimensions, as follows:

$$
\sigma=\frac{\mathrm{L}}{\mathrm{RA}},
$$


$\sigma$ is the ionic conductivity, $\mathrm{R}$ is the electrical resistance, $\mathrm{L}$ is the thickness, and $\mathrm{A}$ is the surface area of the sample. The ionic conductivities of the glass samples were determined in the temperature range $650-750 \mathrm{~K}$. At temperatures lower than $650 \mathrm{~K}$, the impedance of the samples fell out of the upper limit accuracy of the equipment. On the other hand, at temperatures higher than $750 \mathrm{~K}$, the glass approaches its $\mathrm{T}_{\mathrm{g}}$, changing the dependence of the conductivity on temperature.

Finally, the ionic conductivity dependence on the inverse of temperature is plotted by applying the linearized form of the Arrhenius-like relation expressed by: ${ }^{29}$

$$
\sigma \mathrm{T}=\mathrm{A}_{\mathrm{c}} \exp \left(\frac{-\mathrm{E}_{\mathrm{a}}}{\mathrm{kT}}\right)
$$

where $A_{c}$ is the pre-exponential factor, $E_{a}$ is the activation energy for ionic conductivity, $k$ is the Boltzmann constant, and $\mathrm{T}$ is the absolute temperature. Following this methodology, the linear fit slope is proportional to the activation energy, which provides access to the energetic barrier for ionic conductivity. Figure 4 exhibits the Arrhenius-like plots of ionic conductivity obtained by post-mortem impedance spectroscopy of the glass samples synthesized at $1273 \mathrm{~K}$ during different synthesis durations. Irrespective of the synthesis duration and consequently the cerium speciation, the slopes of the linear fits are nearly the same. The calculated activation energy for ionic conductivity in all samples ranged from 1.018 to $1.022 \mathrm{eV}$ and within errors originating from the linear fitting procedure, evidencing that under these conditions, the change in cerium speciation at the used concentration $\left(0.1 \mathrm{wt} \% \mathrm{Ce}_{2} \mathrm{O}_{3}\right)$ had no significant effect on the electrical conductivity of the studied glasses.

By employing the linear fit curves in Figure 4, one can now estimate the conductivity at any fixed temperature. Then, the electrical conductivity can be used to infer the bubble volume fraction 
employing the Maxwell-Wagner equation derived from the "effective medium theory." 22 This model is originally derived for complex conductivity, but it can be easily reduced to the real part of conductivity. The expression derived from this model and adapted for the present case is given by:

$$
\frac{\sigma_{\text {bubbled }}}{\sigma_{\text {bubble-free }}}=\frac{2 \sigma_{\text {bubble-free }}+\sigma_{\text {gas }}-2 \phi_{\mathrm{b}}\left(\sigma_{\text {bubble-free }}-\sigma_{\text {gas }}\right)}{2 \sigma_{\text {bubble-free }}+\sigma_{\text {gas }}+\phi_{\mathrm{b}}\left(2 \sigma_{\text {bubble-free }}-\sigma_{\text {gas }}\right)},
$$

$\sigma_{\text {bubbled }}, \sigma_{\text {bubble-free, }}$ and $\sigma_{\text {gas }}$ are the electrical conductivities of the bubble-glass, free-bubble, and gaseous phases, respectively. $\phi_{\mathrm{b}}$ is the bubble volume fraction of the analyzed bubble-glass sample. By neglecting the electrical conductivity of the gaseous phase, equation (6) is reduced to:

$$
\frac{\sigma_{\text {bubbled }}}{\sigma_{\text {bubble-free }}}=\frac{2-2 \phi_{\mathrm{b}}}{2+\phi_{\mathrm{b}}}
$$

Because the activation energies of all samples are the same, the choice of temperature for lowtemperature measurements is not essential. Therefore, the middle-range temperature $(700 \mathrm{~K})$ was chosen to extract each sample's conductivity, and by applying equation (7), the bubble volume fractions were obtained. Figure 5 presents the overall electrical conductivity and the respective bubble fractions of all glass samples synthesized at $1273 \mathrm{~K}$. The electrical conductivity of the glass samples decreased as a function of the synthesis dwell time up to approximately $60 \mathrm{~min}$ when this tendency was inverted. Conversely, the bubble volume fraction increased for short synthesis durations, but after $60 \mathrm{~min}$, the bubble volume fraction decreased with the synthesis time. In summary, the bubble fraction results were in fair agreement with the density measurements presented above.

\subsection{High-temperature ionic conductivity}


Once bubble volume fraction was sensibly detected by post-mortem electrical conductivity measurements and showed that over time cerium reduction did not play a significant role in the glass matrix's electrical conductivity, we obtained results employing impedance spectroscopy at high temperature, which are presented herein. In this section, the sample from the second group pre-synthesized at $973 \mathrm{~K}$ was used. This sample was evaluated by an in situ technique using hightemperature impedance spectroscopy during the synthesis procedure. Thus, the bubble fraction evolution in the melt can be described as follows: During the measurement, bubbles grow or shrink and escape from the melt, causing changes in its overall electrical conductivity and, consequently, its electrical resistance. The melt impedance response also exhibits a typical ionconducting behavior according to the straight and steep increase of the imaginary part of the impedance (spike) at low frequencies. Nevertheless, as opposed to a glass ion-conducting behavior, owing to the high mobility and thermal vibration of species in the melt, the melt's impedance response does not show any capacitive response at higher frequencies, as expected (Fig. S2). As previously done, an equivalent circuit specific to an ionic liquid was employed to fit the impedance data and extract the resistance of the sample (Fig. S2). ${ }^{25}$

Generally, the electrical conductivity is accessed by determining the shape factor of the cell (L/A ratio). This procedure is performed using an aqueous $\mathrm{KCl}$ solution of known conductivity, which is often demanding work. ${ }^{25}$ However, in this case, we are interested in the ratio between the bubbled glass and the free-bubbled glass conductivities. Thus, the melt resistance is sufficient to determine the bubble volume fraction because the shape factor of the cell does not change during the in situ impedance measurements. Consequently, by rearranging the Maxwell-Wagner model and adapting it to the resistance, using equation (4), one can directly access the bubble volume fraction without determining the cell's shape factor. In this case, an adapted form of the MaxwellWagner model is employed, as follows: 


$$
\frac{\mathrm{R}_{\text {bubble-free }}}{\mathrm{R}_{\text {bubble }}}=\frac{2+\phi_{\mathrm{b}}}{2-2 \phi_{\mathrm{b}}}
$$

Figure 6 presents the evolution of both the resistance and bubble volume fraction by applying the model mentioned above. Both quantities behave similarly because the electrical resistance and bubble volume fraction are directly proportional properties, giving rise to curves that exhibit roughly the same behavior. Indeed, both the resistance and bubble volume fraction increase initially; they pass through a maximum after approximately 20 min of measurements and decrease from this point. In summary, the bubble volume fraction evaluated by this current method is similar to that determined by apparent density and low-temperature impedance spectroscopy methods.

\subsection{Comparison of all investigated methods}

A comparison among the bubble volume fractions, evaluated by three different methods, is presented in this section. Figure 7 compares the bubble volume fraction results obtained by postmortem apparent density, post-mortem low-temperature impedance spectroscopy, and in situ high-temperature impedance spectroscopy. Considering the bubble volume fraction's overall behavior with time for the three methods, the sensitivity of the proposed electrical property-based methods is satisfactory. Furthermore, we can obtain an insight into the initial increase in the bubble fraction in the early stages of bubble fraction evolution. Because a maximum has been observed in all applied methodologies, this behavior is unlikely to be only an artifact of measurements, as suggested by us in a previous study. ${ }^{7}$ In contrast, this maximum should be related to physico-chemical phenomena intrinsic to the system. The initial increase in the bubble fraction might be related to the nucleation of new bubbles or simple gas diffusion to the already formed ones due to the time dependence of cerium incorporation and redox reaction. ${ }^{7}$ 
Discrepancies are spotted, but they can be rationalized based on the intrinsic differences between the methods. First, the in situ high-temperature impedance method seems to have the bubble volume fraction peak shifted when compared to the post-mortem methods. This shift should be expected because the in situ high-temperature method takes between 20 and $30 \mathrm{~min}$ to reach the temperature of measurement $(1273 \mathrm{~K})$ because of the limiting heating rate of the high-temperature impedance system. This enabled the evolution of the bubble fraction before the beginning of the resistance measurements. On the other hand, it has been observed that samples similar to the postmortem methodologies take approximately $3 \mathrm{~min}$ to reach thermal equilibrium when introduced from room temperature in the hot furnace. ${ }^{30}$ The second and more critical discrepancy is the larger values for the post-mortem bubble volume fraction compared to the in situ values. These discrepancies can arise from a series of fundamental differences between post-mortem and in situ methodologies. The most likely, in our view, is linked to a quenching effect on post-mortem samples. It is well known that thermodynamic properties such as volume and even kinetic quantities such as $T_{\mathrm{g}}$ change considerably with the cooling rate. ${ }^{31,32}$ For example, this fundamental idea is used in industrial processes, such as tempered glass production, to create toughened glass sheets. In this process, the surface of the glass is rapidly frozen, defining its apparent volume, while the center experiences much slower cooling rates. This phenomenon results in a smaller equilibrium volume at the center of the piece that cannot be fully accomplished, creating compression and tensile stress in the surface and bulk, respectively. ${ }^{33}$ Similarly, the same occurs in the quenched samples because the outer layer of glass is defined before the center is cooled. However, in this case, the stress created by unmatched volumes would be partially relieved by expanding the bubbles in the bulk before freezing the inner supercooled liquid into a glass. Consequently, the post-mortem samples presented a higher bubble volume fraction than the samples studied directly in the liquid state.

In summary, the in situ impedance spectroscopy method can be used to infer the bubble volume fraction. Moreover, it has a much easier and faster pre-synthesis step, and no further sample preparation steps are required. In addition, it provides in situ information, and in this way, the 
idea of bubble/glass contractions during the cooling stage is not ignored, as it is for the postmortem methods.

\section{CONCLUSION}

Three different methods are used to infer bubble volume fraction in bubbled glasses/melts in this work. These techniques are based on the same reasoning: comparing a particular property of the bubble-glass/melt and converting this property to the corresponding bubble volume fraction. In this study, an in situ impedance spectroscopy method is proposed, tested, and validated to infer the bubble volume fraction in melts. This is done by comparing the proposed methodology with two well-established methods: post-mortem overall density and post-mortem low-temperature impedance spectroscopy.

The in situ bubble fraction determination was performed by impedance spectroscopy, and subsequently, the estimated electrical resistance was converted to the bubble volume fraction using the Maxwell-Wagner model. Globally, the methods studied herein present a similar bubble volume fraction evolution. Slightly lower values for the bubble volume fraction are found for the case of in situ impedance spectroscopy compared with the post-mortem results. However, these discrepancies could be rationalized based on the fundamental differences between the postmortem and in situ methods.

The main results demonstrate the technical viability of this approach. Indeed, the proposed method has a great advantage when compared to post-mortem methods. A much easier presynthesis step and no sample preparation were required. Thus, the time-consuming and laborious characteristics of post-mortem approaches are avoided. In addition, information is acquired in situ at high temperatures, which enables us to avoid the influence of the cooling stage.

\section{ACKNOWLEDGMENTS}


Luiz Pereira is grateful to the Commissariat à l'énergie atomique et aux énergies alternatives (CEA) for his PhD scholarship. We thank Sylvain Mure for the technical support provided during the impedance spectroscopy experiments. We also thank Dr. Maxime Fournier for his contributions and advice.

\section{REFERENCES}

1. Cannillo V, Boccaccini AR. 12 - Finite element modelling of brittle matrix composites. In: Soutis C, Beaumont PWR, editors. Multi-Scale Modelling of Composite Material Systems: Woodhead Publishing; 2005. p. 356-73.

2. Boccaccini AR, Veronesi P, Leonelli C. Microwave processing of glass matrix composites containing controlled isolated porosity. Journal of the European Ceramic Society. 2001 2001/08/01/;21(8):1073-80.

3. Cable M. A century of developments in glassmelting research. Journal of the American Ceramic Society. 1998;81(5):1083-94.

4. Sauvage E, Gagnoud A, Fautrelle Y, Brun P, Lacombe J. Thermoconvective flow of molten glass heated by direct induction in a cold crucible. Magnetohydrodynamics. 2009 (4):53542.

5. Lautze NC, Sisson TW, Mangan MT, Grove TL. Segregating gas from melt: an experimental study of the Ostwald ripening of vapor bubbles in magmas. Contributions to Mineralogy and Petrology. 2011 2011/02/01;161(2):331-47. 
6. Gardner JE, Hajimirza S, Webster JD, Gonnermann HM. The impact of dissolved fluorine on bubble nucleation in hydrous rhyolite melts. Geochimica et Cosmochimica Acta. 2018 2018/04/01/;226:174-81.

7. Pereira L, Podda O, Fayard B, Laplace A, Pigeonneau F. Experimental study of bubble formation in a glass-forming liquid doped with cerium oxide. Journal of the American Ceramic Society. 2020;103(4):2453-62.

8. Lopez-Sanchez MA, Llana-Fúnez S. An extension of the Saltykov method to quantify 3D grain size distributions in mylonites. Journal of Structural Geology. 2016 2016/12/01/;93:149-61.

9. Saltykov S. The Determination of the Size Distribution of Particles in an Opaque Material from a Measurement of the Size Distribution of Their Sections. Stereology. 1967:163 - 73.

10. Lopez-Sanchez M. GrainSizeTools: a Python script for grain size analysis and paleopiezometry based on grain size. The Journal of Open Source Software. 2018 10/06;3:863.

11. Harris WH, Guillen DP, Klouzek J, Pokorny R, Yano T, Lee S, et al. X-ray tomography of feed-to-glass transition of simulated borosilicate waste glasses. Journal of the American Ceramic Society. 2017 Sep;100(9):3883-94. PubMed PMID: WOS:000409281400008. English.

12. Gouillart E, Toplis MJ, Grynberg J, Chopinet M-H, Sondergard E, Salvo L, et al. In Situ Synchrotron Microtomography Reveals Multiple Reaction Pathways During Soda-Lime Glass Synthesis. Journal of the American Ceramic Society. 2012;95(5):1504-7.

13. King A, Guignot N, Deslandes JP, Pelerin M, Joosten I, De Looff D, et al. Recent Tomographic Imaging Developments at the PSICHE Beamline. Integrating Materials and Manufacturing Innovation. 2019 2019/12/01;8(4):551-8. 
14. Auracher H, Daubert J, editors. A capacitance method for void fraction measurements in two-phase flow. Proc 2nd Int Conf Multi-Phase Flow London, BHRA; 1985.

15. Andreussi P, Di Donfrancesco A, Messia M. An impedance method for the measurement of liquid hold-up in two-phase flow. International Journal of Multiphase Flow. 1988 1988/11/01/;14(6):777-85.

16. Nuernberg RB, Bello TS, Fokin VM, Zanotto ED, Rodrigues ACM. Non-stoichiometric crystallization of $\mathrm{Li} 2 \mathrm{SiO} 3$-CaSiO 3 glasses: Residual glass composition from ionic conductivity. Journal of Non-Crystalline Solids. 2019;510:158-65.

17. Prado MO, Campos Jr AA, Soares PC, Rodrigues ACM, Zanotto ED. Liquid-liquid phase separation in alkali-borosilicate glass: An impedance spectroscopy study. Journal of NonCrystalline Solids. 2003;332(1-3):166-72.

18. Ravagnani C, Keding R, Rüssel C. High temperature impedance spectroscopy of homogeneous and phase separated melts and glasses of the composition $48.5 \mathrm{SiO} 2 \cdot 48.5 \mathrm{~B}$ 2O3·3Na2O. Journal of Non-Crystalline Solids. 2003;328(1-3):164-73.

19. Bragatto CB, Cassar DR, Peitl O, Souquet JL, Rodrigues ACM. Structural relaxation in AgPO3 glass followed by in situ ionic conductivity measurements. Journal of Non-Crystalline Solids. 2016;437:43-7.

20. Plodinec J. Borosilicate Glasses for Nuclear Waste Immobilization. Glass Technology European Journal of Glass Science and Technology Part A. 2000 12/01;41:186-92. 
21. Pinet O, Phalippou J, Di Nardo C. Modeling the redox equilibrium of the Ce4+/Ce3+ couple in silicate glass by voltammetry. Journal of Non-Crystalline Solids. 2006;352(5051):5382-90.

22. Barsoukov E, Macdonald JR. Impedance Spectroscopy: Theory, Experiment, and Applications2005. 1-595 p.

23. Pflieger R, Malki M, Guari Y, Larionova J, Grandjean A. Electrical Conductivity of RuO2-Borosilicate Glasses: Effect of the Synthesis Route. Journal of the American Ceramic Society. 2009 06/01;92:1560-6.

24. Cawthorne CD, Sinclair WJ, editors. An apparatus for density determination on very small solid samples1972: Journal of Physics E: Scientific Instruments.

25. Simonnet C, Phalippou J, Malki M, Grandjean A. Electrical conductivity measurements of oxides from molten state to glassy state. Review of Scientific Instruments. 2003;74(5):280510.

26. Mekki A. X-ray photoelectron spectroscopy of CeO2-Na 2O-SiO2 glasses. Journal of Electron Spectroscopy and Related Phenomena. 2005;142(1):75-81.

27. Deshpande VK, Taikar RN. Effect of cerium oxide addition on electrical and physical properties of alkali borosilicate glasses. Materials Science and Engineering: B. 2010 2010/08/15/;172(1):6-8.

28. Jayasinghe GDLK, Dissanayake MAKL, Bandaranayake PWSK, Souquet JL, Foscallo D. Electronic to ionic conductivity of glasses in the Li2O-V2O5-TeO2 system. Solid State Ionics. 1999;121(1):19-23. 
29. Nuernberg RB. Numerical comparison of usual Arrhenius-type equations for modeling ionic transport in solids. Ionics. 2020;26(5):2405-12.

30. Pereira L, Kloužek J, Vernerová M, Laplace A, Pigeonneau F. Experimental and numerical investigations of an oxygen single-bubble shrinkage in a borosilicate glass-forming liquid doped with cerium oxide. Journal of the American Ceramic Society. 2020;103(12):673645 .

31. Zheng Q, Zhang Y, Montazerian M, Gulbiten O, Mauro JC, Zanotto ED, et al. Understanding Glass through Differential Scanning Calorimetry. Chemical Reviews. 2019 2019/07/10;119(13):7848-939.

32. Vollmayr K, Kob W, Binder K. How do the properties of a glass depend on the cooling rate? A computer simulation study of a Lennard-Jones system. The Journal of Chemical Physics. 1996;105(11):4714-28.

33. Lee EH, Rogers TG, Woo TC. Residual Stresses in a Glass Plate Cooled Symmetrically from Both Surfaces. Journal of the American Ceramic Society. 1965;48(9):480-7. 
FIGURE CAPTIONS

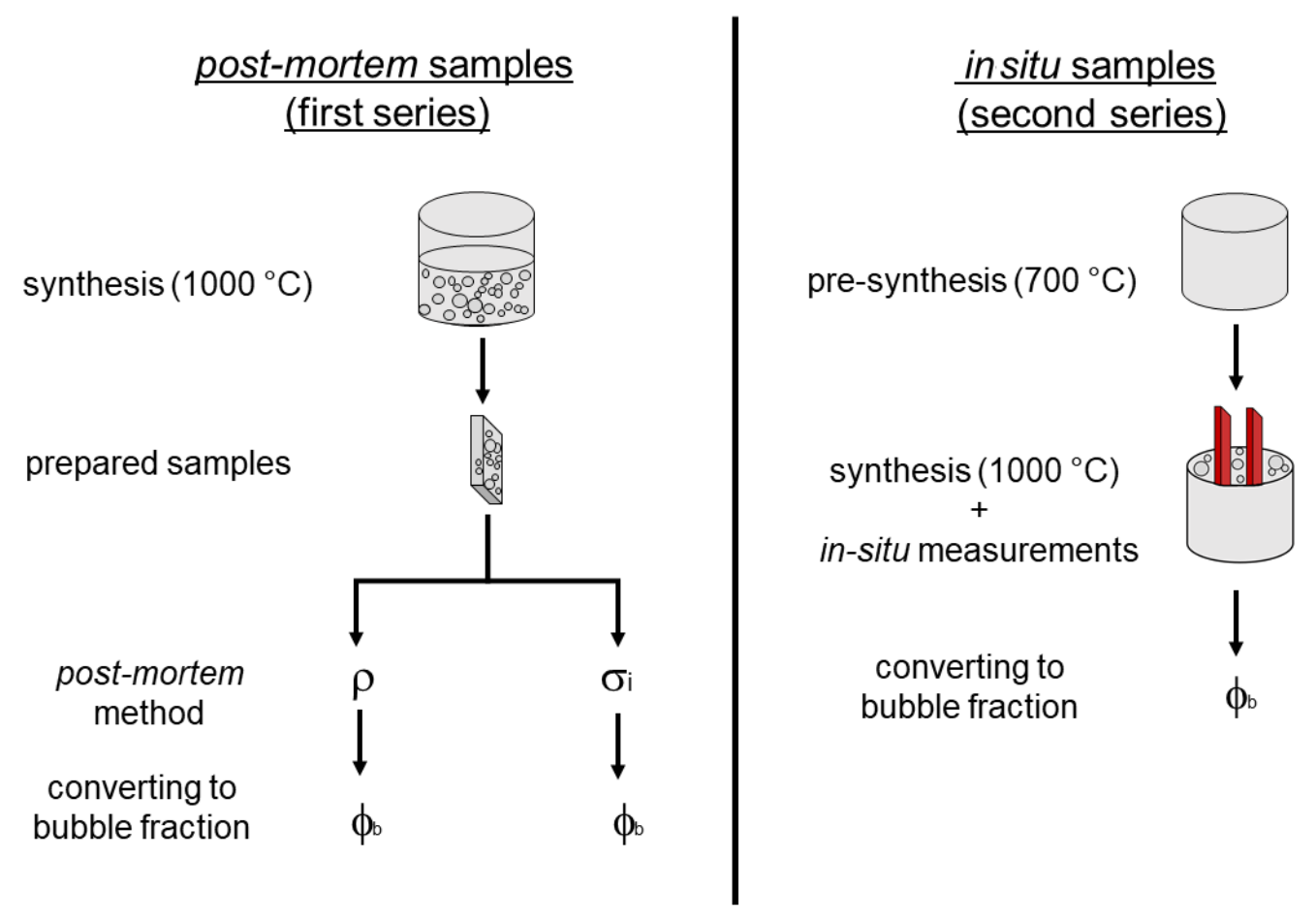

Figure 1: Scheme illustrating the post-mortem and in situ samples synthesized in this work along with their respective bubble investigation methodology. 


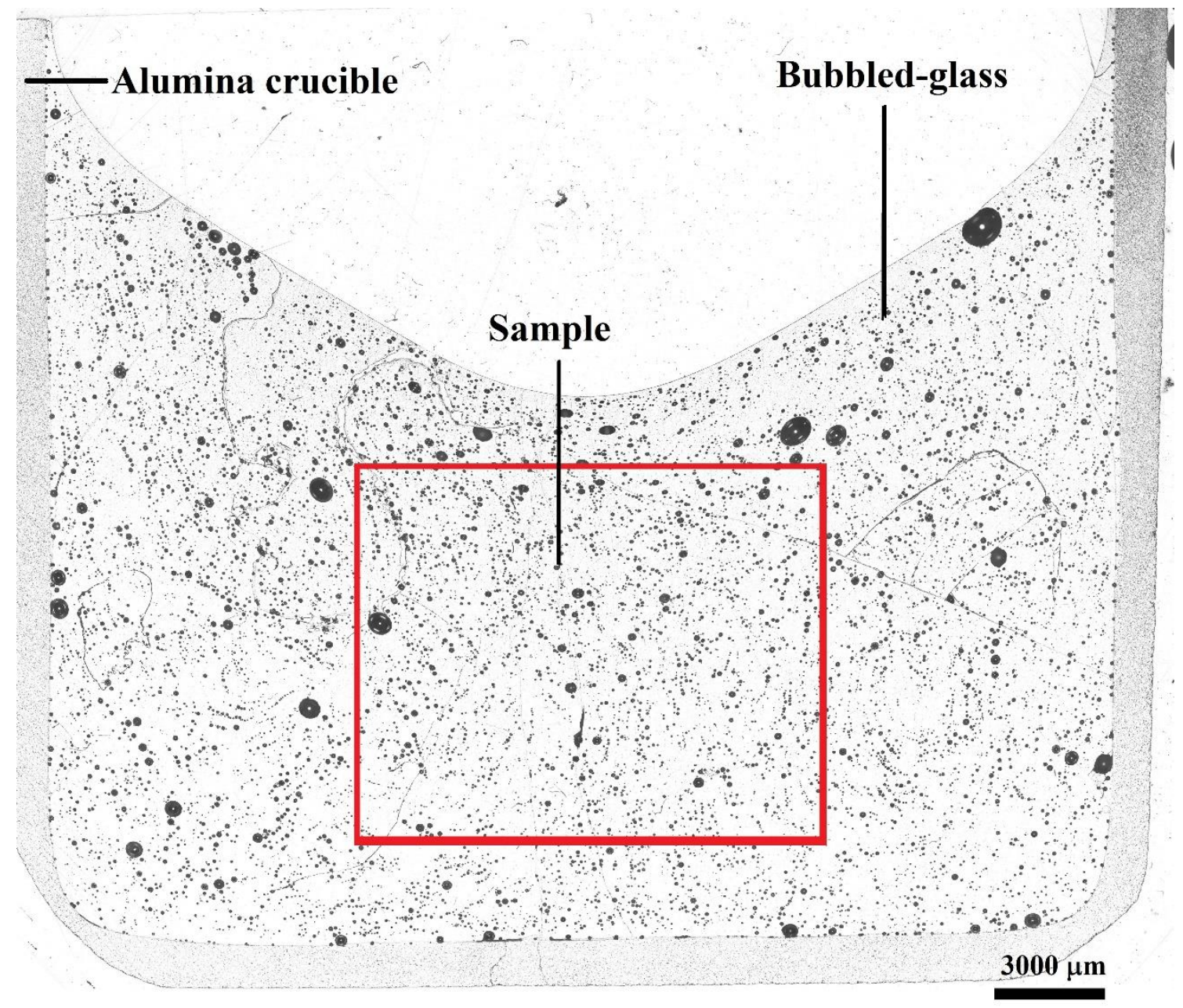

Figure 2: Optical microscopic image of the half-cut crucible containing the glass synthesized at $1273 \mathrm{~K}$ during 20 min showing schematically the parallelepiped sample used for density and lowtemperature impedance spectroscopy measurements. 


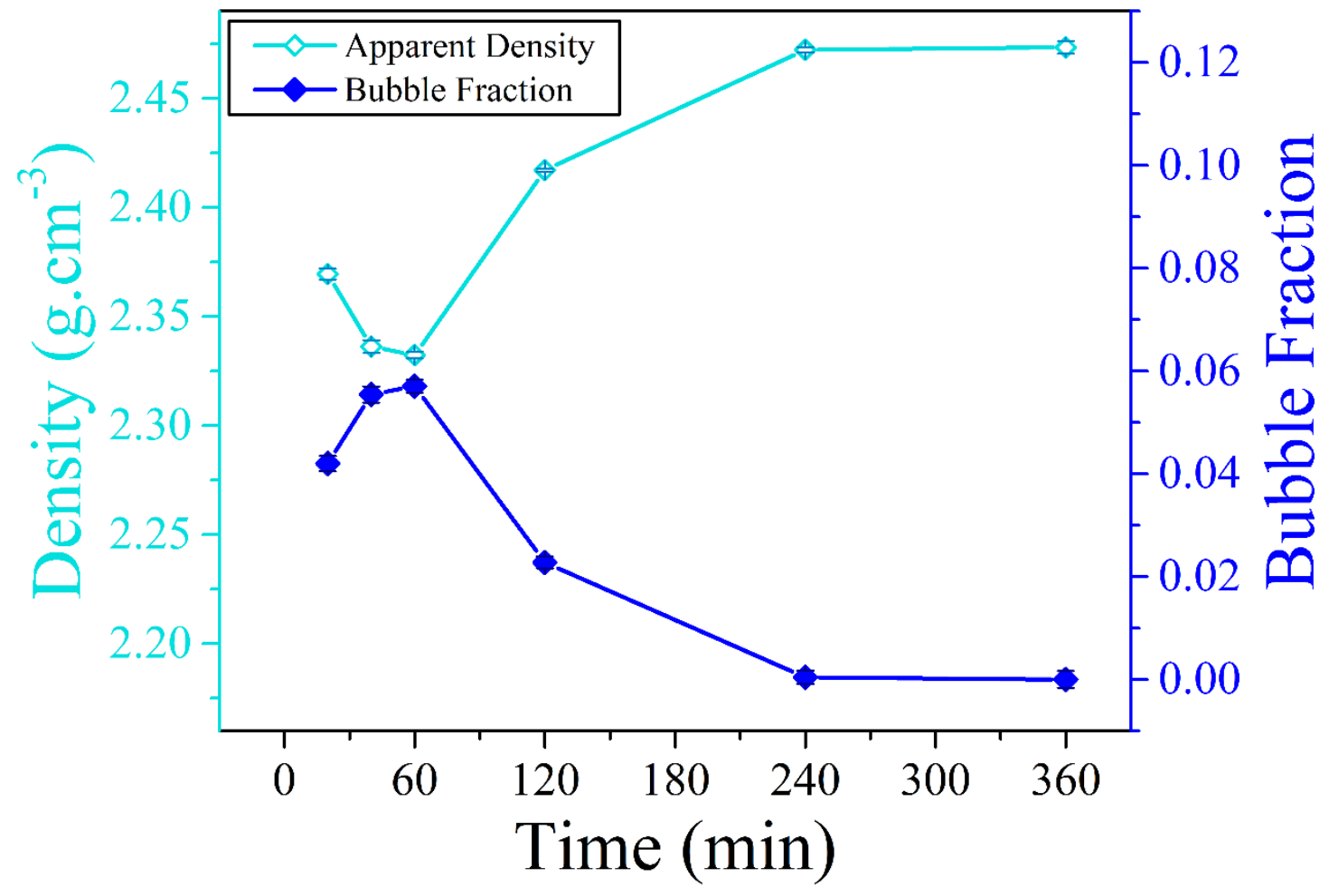

Figure 3: Apparent density and volume bubble fraction of glasses having different synthesis durations at $1273 \mathrm{~K}$. The standard errors are calculated based on three weightings of the same sample but, in most of the cases, the error bars are smaller than the symbols. 


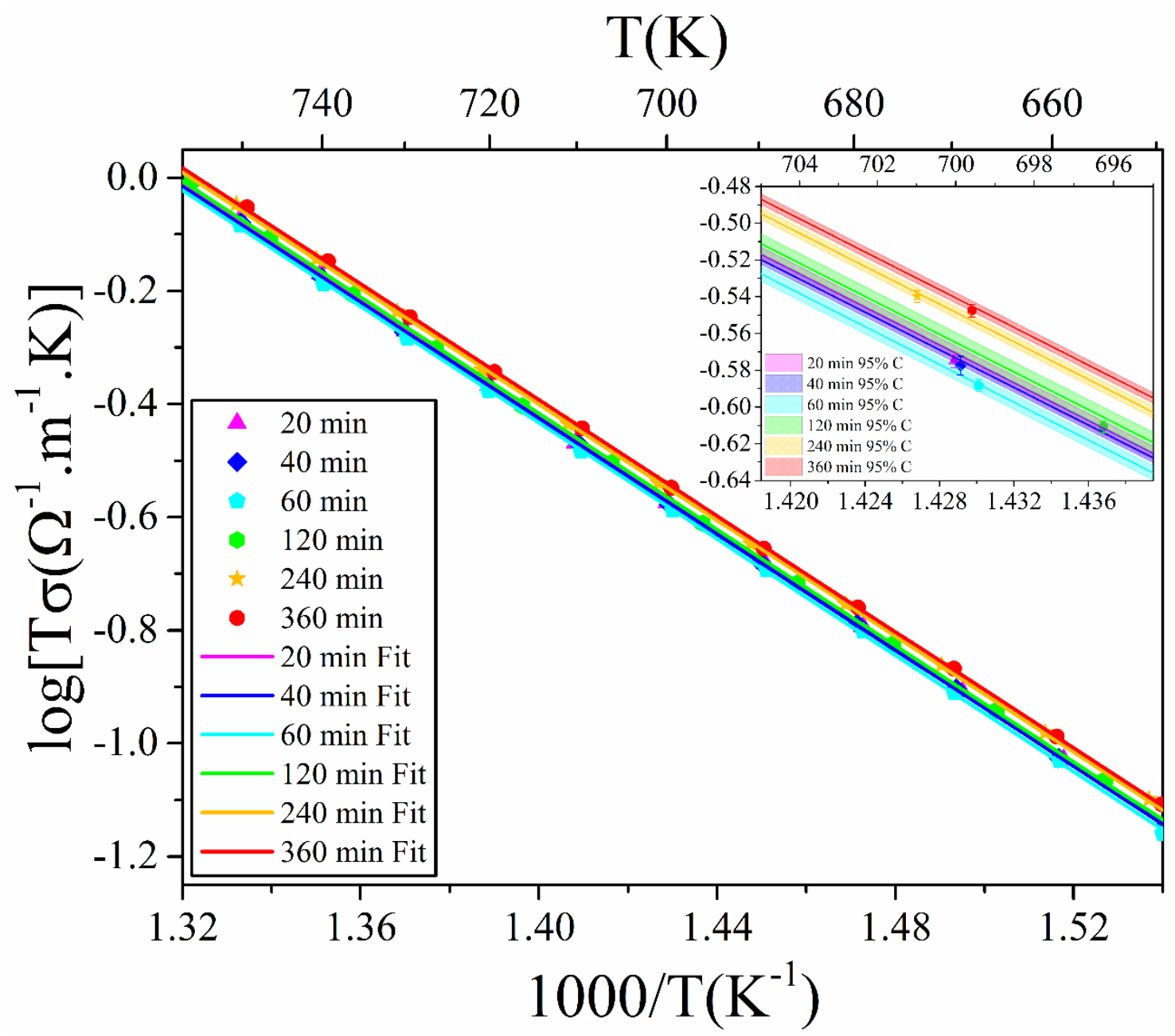

Figure 4: Arrhenius-like plots of the ionic conductivity of glasses synthesized under distinct dwell time and consequently having different bubble fractions and cerium speciation. The confidence interval $(95 \%)$ is also detailed on the zoomed image. 


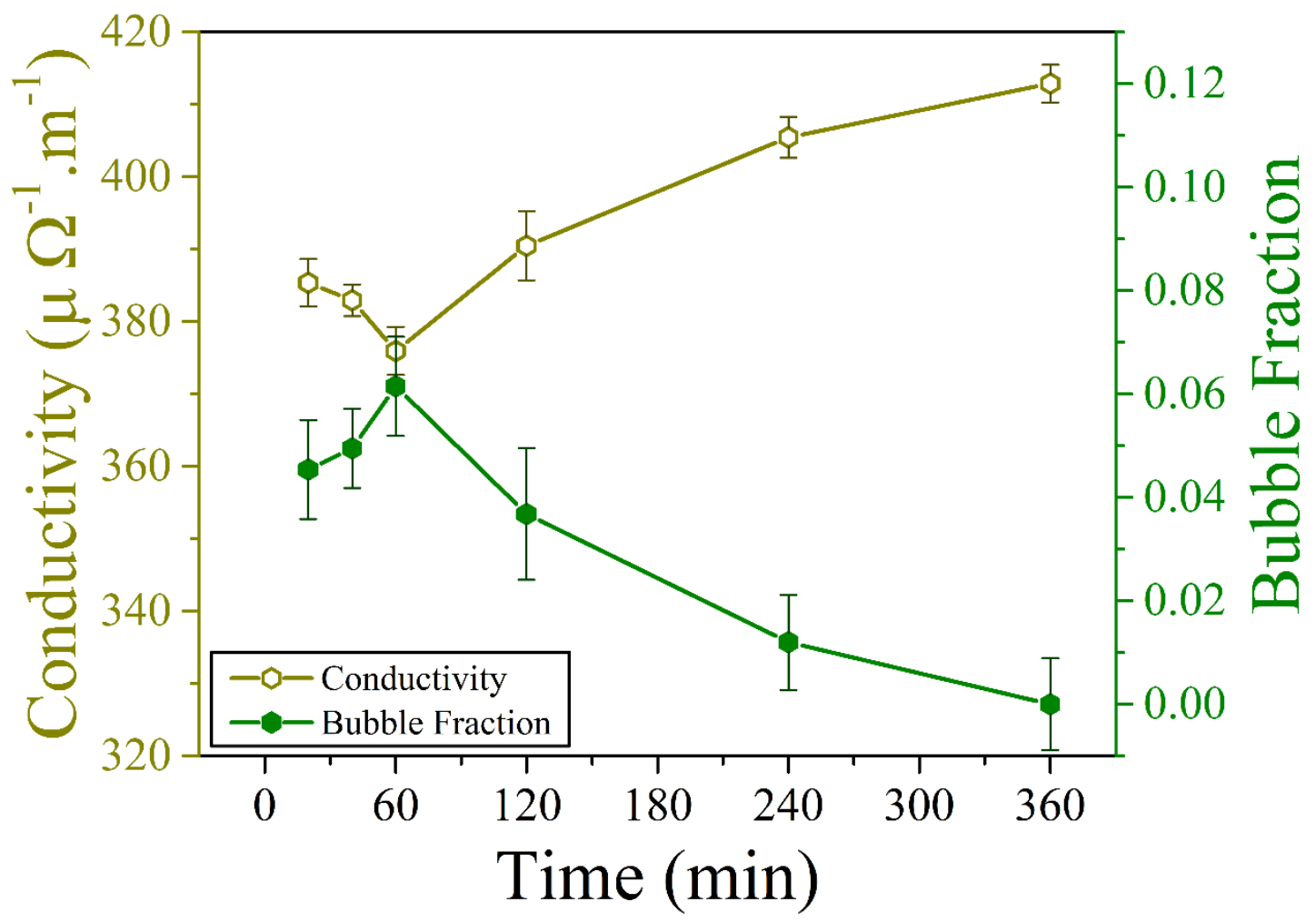

Figure 5: Electrical conductivity (at $700 \mathrm{~K}$ ) and the respective bubble volume fraction of glass samples synthesized at $1273 \mathrm{~K}$ for different synthesis durations. The standard errors presented here are propagated from the $95 \%$ confidence bands sourced from the Arrhenius linear fits. 


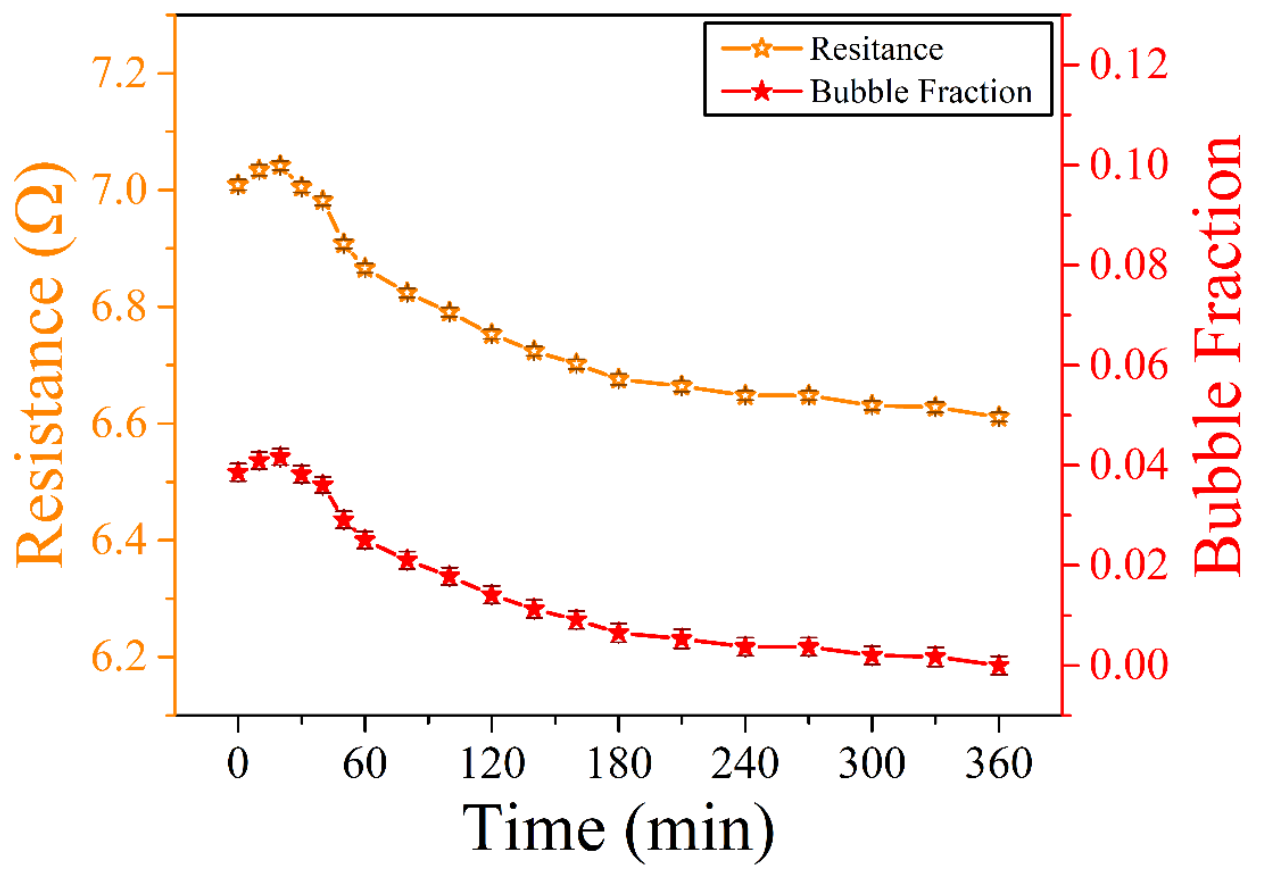

Figure 6: Evolution of the electrical resistance, obtained by high-temperature impedance spectroscopy, and the respective bubble volume fraction versus time for the Ce-doped molten sample synthesized at $1273 \mathrm{~K}$. 


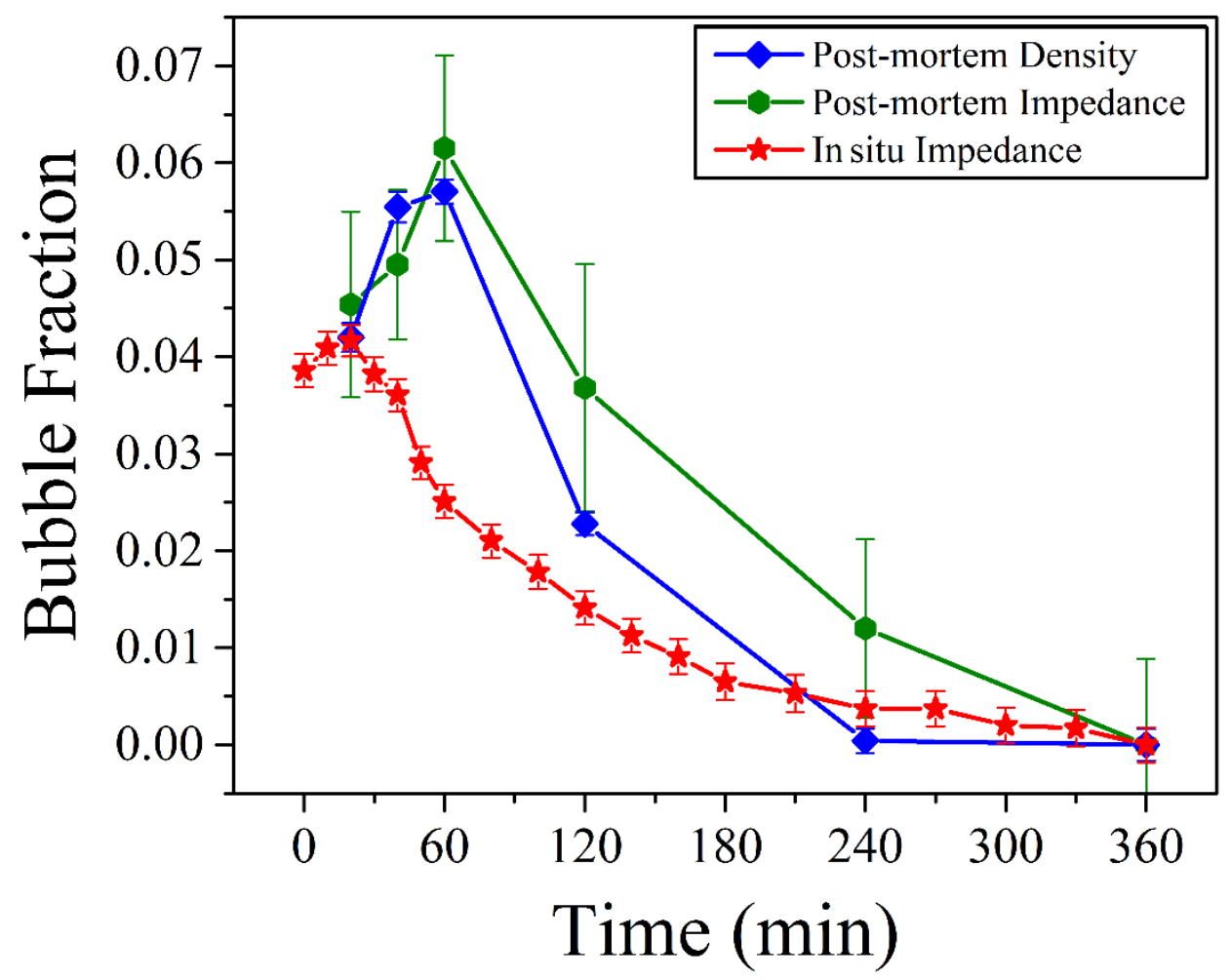

Figure 7: Bubble volume fraction $\left(\phi_{\mathrm{b}}\right)$ evaluated by the three studied methods: post-mortem apparent density measurements and low-temperature impedance spectroscopy as well as in situ high-temperature impedance spectroscopy. 

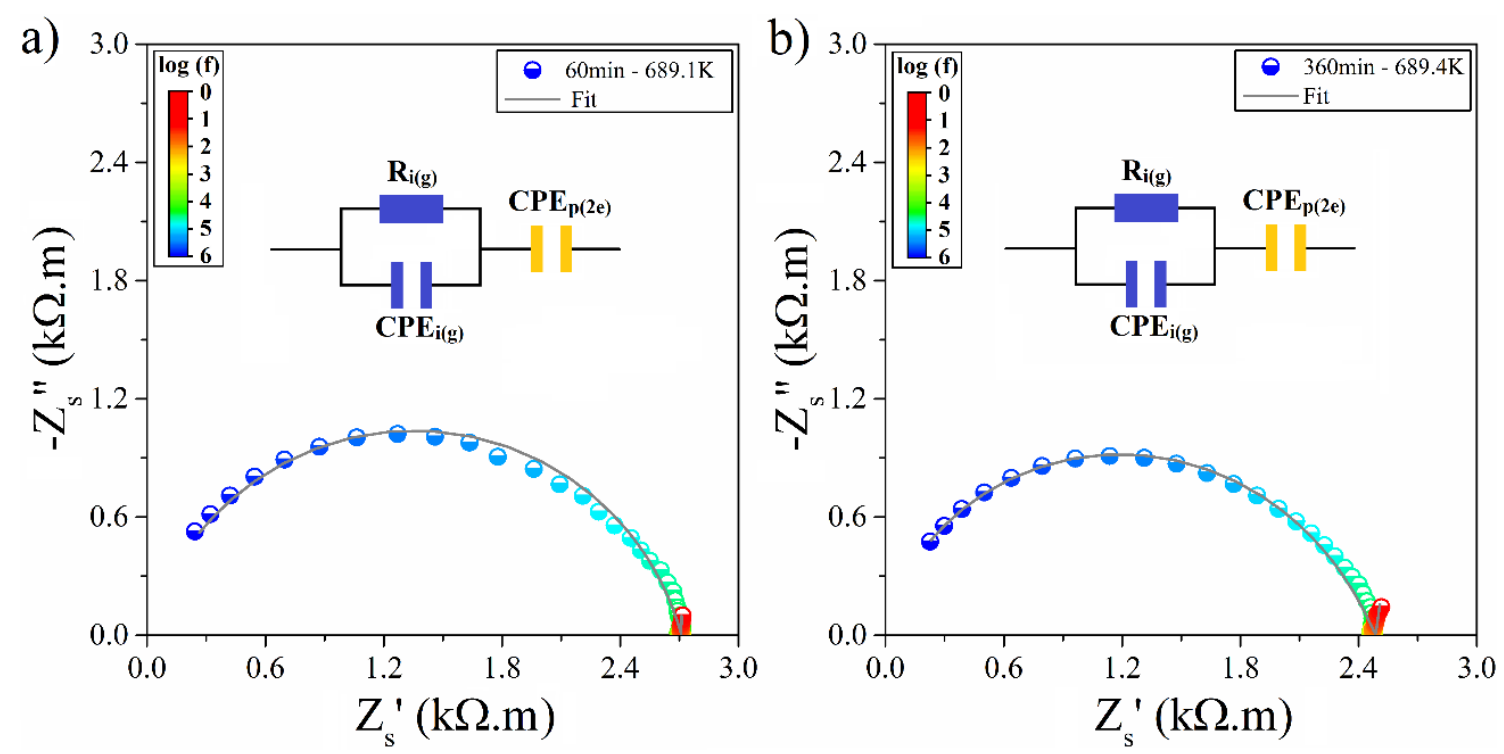

Figure S1: Typical complex impedance plots of Ce-doped glasses synthesized during a) $60 \mathrm{~min}$ and b) $360 \mathrm{~min}$. Measurements are performed under $\mathrm{T}_{\mathrm{g}}$ in the low-temperature impedance measurement system. The complex impedance data shown here have already been normalized regarding the shape factor of each sample for comparison purposes. The shown equivalent circuits are employed for impedance data fitting. Acronyms R and CPE represent resistive and constant phase elements. I and $\mathrm{p}$ denote ionic and polarization mechanisms; $\mathrm{g}$ and $2 \mathrm{e}$ account for the glass sample response and the 2-electrode setup, respectively. 

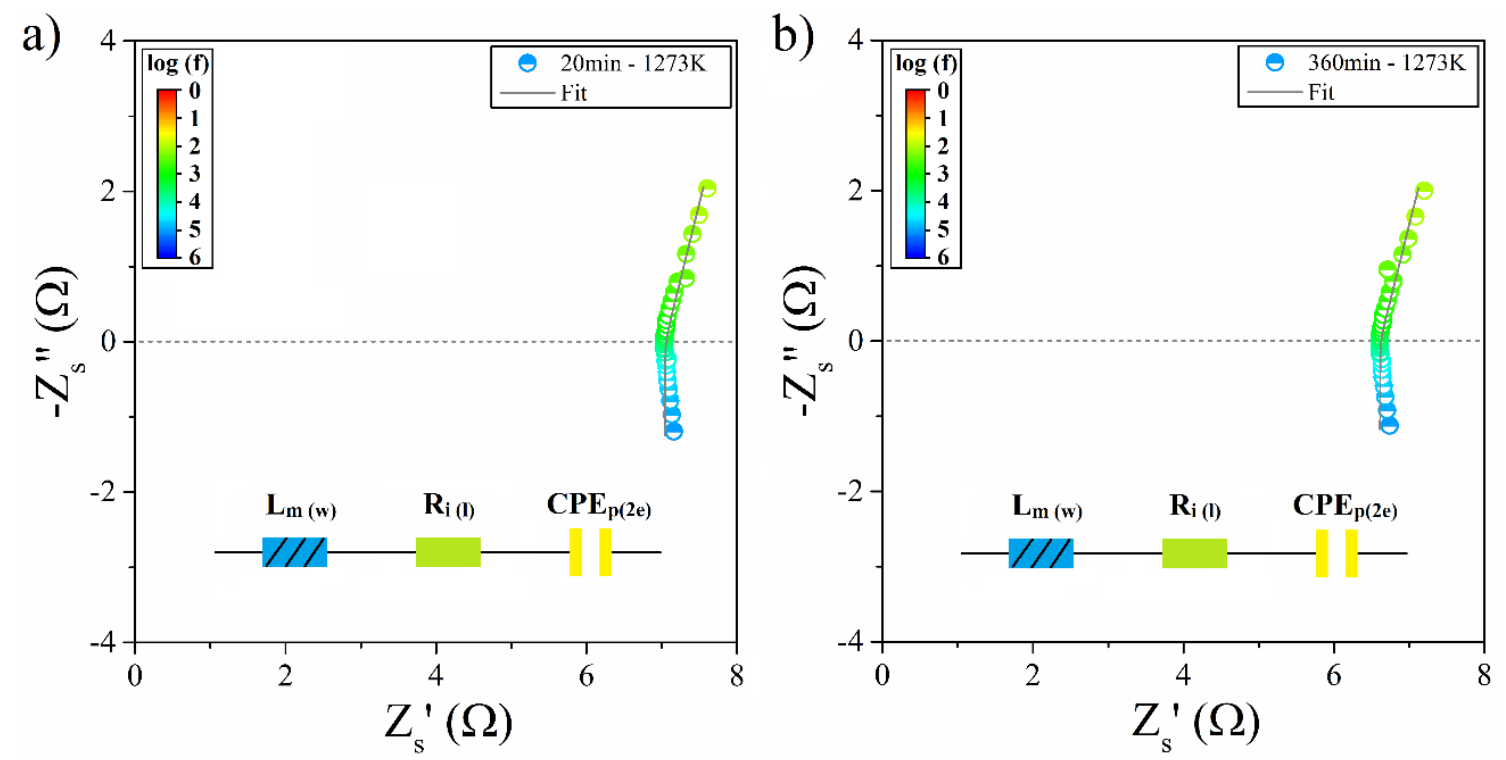

Figure S2: Typical complex impedance plots of Ce-doped glass melts synthesized during a) 20 min and b) $360 \mathrm{~min}$. Measurements are performed above $\mathrm{T}_{\mathrm{g}}$ in the high-temperature impedance measurement system. The shown equivalent circuits are employed for impedance data fitting. Acronyms L, R, and CPE represent inductive, resistive, and constant phase elements, respectively. $\mathrm{i}$ and $\mathrm{p}$ denote ionic and polarization mechanisms; $\mathrm{w}, 1$, and 2e account for the cell wires, glassforming liquid sample response, and the 2-electrode setup, respectively. 


\section{TABLES}

Table 1: Glass composition in wt $\%$

(Composition is not dependent on multivalent speciation).

\begin{tabular}{c|c}
\hline Oxide & Glass + 0.1 wt \% $\mathbf{C e}_{2} \mathbf{O}_{3}$ \\
\hline $\mathrm{SiO}_{2}$ & 58.41 \\
\hline $\mathrm{B}_{2} \mathrm{O}_{3}$ & 18.56 \\
\hline $\mathrm{Na} 2 \mathrm{O}$ & 7.00 \\
\hline $\mathrm{CaO}$ & 5.16 \\
\hline $\mathrm{Al}_{2} \mathrm{O}_{3}$ & 3.99 \\
\hline $\mathrm{ZnO} \mathrm{L}$ & 3.24 \\
\hline $\mathrm{Li}_{2} \mathrm{O}$ & 2.50 \\
\hline $\mathrm{ZrO}_{2}$ & 0.62 \\
\hline $\mathrm{K}_{2} \mathrm{O}$ & 0.21 \\
\hline $\mathrm{Ce}_{2} \mathrm{O}_{3}$ & 0.10 \\
\hline $\mathrm{BaO}_{2}$ & 0.10 \\
\hline $\mathrm{TiO}_{2}$ & 0.08 \\
\hline $\mathrm{Fe}_{2} \mathrm{O}_{3}$ & 0.04 \\
\hline $\mathrm{Total}_{3}$ & \\
\hline & \\
\hline & \\
\hline
\end{tabular}

